\title{
Der Klassiker im April: Kommen Sie zum "young dentists meeting“ nach Bonn!
}

Am 8. und 9. April machen sich wieder junge Zahnärzte und Zahnärztinnen aus dem ganzen Bundesgebiet auf den Weg nach Bonn. Denn dort findet das Frühjahrstreffen der young dentists statt. Auf dem Programm der Veranstaltung mit Tradition steht dieses Mal der Weg in die Selbstständigkeit sowie chirurgisches Basiswissen für den Alltag.

\section{Samstag, 8. April, 14.00 Uhr}

Startup Zahnarztpraxis - der Weg in die Selbständigkeit Referentin: Diana Brendel, M.Sc.

Der junge Zahnarzt hat viele Möglichkeiten der Berufsausübung - und genau das macht die Entscheidung so schwer! Ist die Grundsatzentscheidung zur Niederlassung getroffen, muss sich der Zahnarzt zwischen einer Neugründung und der Übernahme einer bestehenden Praxis entscheiden. Abzuwägen ist auch, ob man als „Einzelkämpfer“ oder in einer Gemeinschaft arbeiten möchte.

Bereits bei dieser Entscheidungsfindung spielen wirtschaftliche Aspekte eine wesentliche Rolle. Und ist die Praxis dann eröffnet, so ist man plötzlich nicht mehr nur Zahnarzt, sondern auch Unternehmer. Ab diesem Zeitpunkt ist es unerlässlich, sich mit Managementthemen und insbesondere mit den Finanzen zu beschäftigen, denn ein betriebswirtschaftliches Grundverständnis ist die Voraussetzung für den Erfolg der eigenen Praxis.

In diesem Seminar erhalten Sie wertvolle Tipps zur Praxisgründung, erlernen das Basiswissen der Betriebswirtschaft in der Zahnarztpraxis und bekommen einen Einblick in die Unternehmensführung.

\section{Folgende Themen erwarten Sie:}

$\rightarrow$ Entscheidungshilfen zur Selbstständigkeit

$\rightarrow$ Einzelpraxis vs. Gemeinschaftspraxis

$\rightarrow$ Praxisgründung vs. Praxisübernahme

$\rightarrow$ Fakten vs. Emotionen

$\rightarrow$ Betriebswirtschaftliche Grundlagen

(Gewinn/Rentabilität/Liquidität)

$\rightarrow$ Erfolgreiche Praxisführung

$\rightarrow$ Praxiscontrolling

15.00 Uhr: Fitnesspause auf Einladung der Deutschen Apotheker- und Ärztebank

19.00 Uhr: Gemeinsames Abendessen mit den Referenten

\section{Sonntag, 9. April, 09.00 Uhr}

Besser mit: Medizinisches und chirurgisches Basiswissen im Praxisalltag - mit praktischen Übungen

Referent: Dr. Dr. Markus Tröltzsch

Medizinisches Wissen wird im Rahmen des zahnmedizinischen Studiums nur am Rande vermittelt. Allerdings wird dieses für den Zahnarzt immer wichtiger, da die gesundheitliche Situation der Patienten durch multiple Medikamentengaben und zahnärztlich relevante Erkrankungen immer komplexer wird. Insbesondere für Patienten, bei denen parodontologische oder chirurgische Maßnahmen nötig sind, ist dieses Wissen von Nöten. Zudem werden auch chirurgische Fähigkeiten meist nur minimal vermittelt. Praxisrelevante Kenntnis von Anatomie, Sites, Schnittführung und Material muss somit häufig selbst erworben werden und steht am Anfang häufig als großes Hindernis der chirurgischen Tätigkeit im Weg.

In diesem Kurs soll medizinisches und chirurgisches Basiswissen für den Alltag vermittelt werden und sowohl auf die für den Zahnarzt wichtige Pharmakologie als auch auf häufige Erkrankungen eingegangen werden. In praktischen Übungen werden zudem grundlegende chirurgische Techniken trainiert.

\section{Programm:}

A) Medizinische Grundlagen für die orale Chirurgie

$\rightarrow$ Einführung

$\rightarrow$ Die häufigsten medizinischen Herausforderungen

$\rightarrow$ Pharmakologie

$\rightarrow$ Evaluation von Patienten

B) Chirurgie Basistechniken

$\rightarrow$ Anatomie Grundlagen der Mundhöhle

$\rightarrow$ Material für verschiedene Indikationen

$\rightarrow$ Chirurgische Basistechniken

Ende: etwa 16:30 Uhr

\section{Jetzt buchen!}

Nähere Informationen zu diesem Seminar erhalten Sie natürlich auch im Internet.

Hier können Sie sich auch zu dem Meeting anmelden:

www.young-dentists.de.

Dieses umfangreiche Fortbildungsprogramm bieten wir Ihnen in einem Komplettpaket (inklusive Unterkunft und Verpflegung) zu besonders günstigen Konditionen an:

Registrierte $y d^{2}$-Teilnehmer

+ FVDZ und DGZMK/APW-Mitglied 80,00 EUR

Registrierte $y d^{2}$-Teilnehmer

+ FVDZ-Mitglied

120,00 EUR

Registrierte $y d^{2}$-Teilnehmer

+ DGZMK/APW-Mitglied

Registrierte $y d^{2}$-Teilnehmer 